\title{
Effectiveness of Action Observation Training on Upper Limb Motor Function in Children with Hemiplegic Cerebral Palsy: A Systematic Review of Randomized Controlled Trials
}

This article was published in the following Dove Press journal:

Pediatric Health, Medicine and Therapeutics

\author{
Abayneh Alamer (D) \\ Haimanot Melese (D) \\ Belaynew Adugna ${ }^{2}$ \\ 'Department of Physiotherapy, School of \\ Medicine, College of Health Sciences, \\ Ayder Comprehensive Specialized \\ Hospital, Mekelle University, Mekelle, \\ Ethiopia; ${ }^{2}$ Department of Physiotherapy, \\ School of Medicine, College of Health \\ Sciences, Tibebe Ghion Comprehensive \\ Specialized Hospital, Bahirdar University, \\ Bahirdar, Ethiopia
}

\begin{abstract}
The purpose of this review was to evaluate and examine the current best evidence for the effectiveness of action observation therapy on upper limb function rehabilitation in children with hemiplegic cerebral palsy. A comprehensive search of literature published between September 2010 and May 2020 was conducted using the following electronic databases: PubMed, Google Scholar, the Physiotherapy Evidence Database (PEDro), EMBASE, Cochrane library, and Scopus. Only randomized controlled trials evaluating the effect of action observation therapy on upper limb motor function in children with hemiplegic cerebral palsy were included. PEDro scale was used to assess the risk of bias of included trials. This study was reported according to the guideline of the PRISMA statement. The overall methodological quality of the studies was done using the PEDro scale and GRADE approach. The primary outcome measures of this review were the Melbourne Assessment Scale, Assisting Hand Assessment scale to evaluate physical function and structures. Furthermore, the ABILHAND-Kids test, and Box and Block Test primary outcome measures were used to determine the subjects' activities and participation. Nine randomized controlled trials involving 234 participants were analyzed. The overall quality of evidence was rated from moderate to high. This review suggests action observation therapy was found to be a promising intervention for upper limb rehabilitation in children with cerebral palsy.
\end{abstract}

Keywords: action observation training, hemiplegic CP, upper extremity motor function, systematic review

\section{Introduction}

Cerebral palsy (CP) is a set of permanent movement and posture disorders that causes restriction of activities in the fetal or newborn brain. ${ }^{1}$ It is a major leading cause of disabilities in childhood with a prevalence of 2.1 per 1000 live births, ${ }^{2,3}$ and children with unilateral cerebral palsy (UCP) accounts for $39 \%$ of the general CP population. ${ }^{3,4}$

Often, movement deficits include poor coordination, muscle weakness, tremors, sensory deficits, poor perception, and severe difficulties with a concentration in children with CP. ${ }^{1,5}$ These great burdens of health problems may be associated with restricted motor skills and activities of daily living, such as reaching and grasping, which have a significant impact on their upper limb motor function. ${ }^{1,5-7}$
Correspondence: Haimanot Melese

Tel +25 I 927608383

Fax +25I 3444I668I

Email haimanotphysio@gmail.com 
Nowadays, numerous goal-directed therapeutic approaches have been used to enhance upper limb function, including constraint-induced movement therapy (CIMT), hand-arm bimanual intensive therapy (HABIT), intramuscular botulin toxin combined with therapeutic training, and action observation therapy (AOT). ${ }^{8-10}$ Among these, AOT has recently gained great attention in improving upper limb function. ${ }^{11}$

AOT is a novel rehabilitation technique, which involves observation of purposeful actions with the intention to imitate and then performing those actions. ${ }^{12,13}$ Often time, it is usually described as the patient being asked to carefully observe actions presented through a video-clip or performed by an operator, in order to imitate, try, and execute them after observation. ${ }^{14}$ This may include the mirror neuron system (MNS) processes on the upper limb function resulting from the observation of actions (new motor skills) and actual execution of similar neural structures. ${ }^{15-17}$ Current evidence ${ }^{18-20}$ reported that AOT is becoming the latest treatment option among other interventions for children with UCP on upper limb function and their activities of daily living. Previous reviews have been limited to investigating AOT effectiveness on upper and lower limb rehabilitation in both neurological and orthopedic disorders, ${ }^{11}$ patients with stroke and brain injuries, ${ }^{21-23}$ limb pain, ${ }^{24}$ and in Parkinson disease. ${ }^{25}$

However, despite the volume of our understanding, there is a dearth of current scientific evidence evaluating the efficacy of AOT on upper limb functions, particularly in children with UCP in a systematic way. Given these gaps, there is a need to perform a systematic evaluation to investigate the current scientific evidence on AOT clinical worthiness with high quality randomized controlled trials. Therefore, the purpose of this review was aimed to address the clinical research question about the effectiveness of AOT on upper limb function in children with unilateral CP.

\section{Methods}

\section{Design}

This systematic review was performed and reported in accordance with the Preferred Reporting Items for Systematic Reviews and Meta-Analyses (PRISMA) guidelines. $^{26}$

\section{Search Strategy}

A literature search was performed to recognize all qualified randomized controlled trials. The literature search was conducted using the following databases: PubMed, Google scholar, Cochrane library, Scopus, CINAHL, AMED, PEDro, and EMBASE. The terms used to search the literature were: "Action observation training" OR "Action observation treatment" OR "Action Observation Therapy" AND "upper limb functions" OR "movement execution" OR "physical training" AND "unilateral cerebral palsy" OR "hemiplegic/hemiplegia cerebral palsy" OR "cerebral palsy" AND "randomized clinical trial" (Appendix). The retrieval of the studies for the published articles was set for the period of September 2010 to May 2020. Manual searches of the reference list of included articles were also performed.

\section{Eligibility Criteria}

The studies were included if they met the following established inclusion criteria: 1) population/participants: children with unilateral cerebral palsy; 2) intervention: intervention groups received AOT and/or combined with constraintinduced movement therapy; 3) comparison: control groups received sham AOT/repetitive physical training; 4) outcomes: physical function and structure, activities and participation. All randomized control trials (RCT) performed to evaluate the efficacy of AOT on UCP survivors were included in this review. Studies in which the addition of AOT over other conventional interventions (experimental group) compared with other interventions only (control group) were also included. Only full-length articles reported in English were included. This review excluded observational studies, quasi-experimental studies, conference abstracts, and RCTs which include healthy children, children with stroke, and traumatic brain injuries.

\section{Study Selection}

Two reviewers (H.M, A.A) independently screened papers from the identified lists on the basis of title/abstract, based on the determined inclusion standards. The studies have been retrieved in detail by means of methodological quality and data extraction tools. Reference lists of recognized studies have been manually searched for extra RCTs. The duplicated articles were eliminated. Eligible papers have been collected in complete-textual content, independently screened by the same reviewers. The third reviewer (B.A) was there to resolve the disagreements among the two reviewers.

\section{Risk of Bias in Individual Studies}

The risk of bias of the included studies was independently assessed by two review authors (H.M and A.A) by using the Physiotherapy Evidence Database (PEDro) scale. The 
methodological quality of each included study has been assessed using the PEDro scale. ${ }^{27,28}$ This tool comprises 10 items with the first item (external validity of the article) quality assessments of controlled intervention study tool. ${ }^{26}$ The following topics were assessed based on critical criteria: concealed assignment, intention-to-treat analysis, and adequate treatment follow-up. These characteristics make the PEDro scale a valuable apparatus to evaluate the methodological quality of RCTs. This review considered trials with a score of 5 to 7 as moderate quality, and a score of $\geq 8$ as high quality (Table 1). The overall quality of the evidence and strength recommendation was evaluated by using the GRADE approach. ${ }^{29}$ The GRADE approach specifies four levels of quality (high, moderate, low, and very low). The overall evidence was downgraded depending on the presence of five factors: limitations (due to risk of bias); consistency of results; directness (eg, whether participants are similar to those about whom conclusions are drawn); precision (ie, sufficient data to produce narrow confidence intervals); and other (eg, publication bias).

\section{Data Extraction}

Information extraction was done based on the data retrieval template of the Cochrane Consumer and Communication Review Group. Two reviewers (H.M \& A.A) extracted the data independently and the third author checked the extracted data. Disagreements were resolved by consensus among the two authors. If the two authors did not reach a consensus, the third author (B.A) was there to decide. The following data were extracted from each trial using PICOS standards: participant characteristics (sex, mean age, sample size, duration of the disease), the objective of the study, author name and year of publication, PEDro score, follow-up time and intensity of the training, treatment outcomes (at baseline, follow-up, and end-of-treatment), type of treatment, study design, study results, and study conclusions (Table 2).

\section{Results}

\section{Study Selection}

A total of 2507 articles were identified from databases by the searching strategy. After adjusting for duplicates, 1882 remained. After the title and abstract screening among 644 studies, 594 studies were excluded. After full-text screening out of 50 articles, 9 randomized controlled trials were included in this review (Figure 1).

\section{Study Characteristics}

The detailed descriptions, and characteristics of the included studies are summarized in Table 1. All nine randomized controlled trials, which focused on the effectiveness of AOT in the treatment of the upper limb function of children with hemiplegic cerebral palsy, were included in the current systematic review.

\section{Participants}

All subjects who participated in individual studies had a clinical diagnosis of hemiplegic cerebral palsy (CP). The sample size ranged from $10^{30}$ to 59 participants. ${ }^{31}$ The mean age (SD) of participants ranged from $9.13(2.36)^{32}$ to 9.48 $(2.12)^{20}$ years in experimental groups and $9.25(3.15)^{32}$ to $9.94(3.15)^{20}$ years in controlled groups.

\section{Interventions}

Randomized controlled trials were included that assessed the effectiveness of AOT combined or not with mCIMT, compared with a comparison/control group; assessed sham/placebo AOT (watching videos of computer games and landscape photographs with no specific motor content); and/routine conventional rehabilitation programs. The training durations were ranged from 20 minutes to 1 hour per session, 1 up to 5 times per week, for three weeks to six months. ${ }^{18,20,33}$ The characteristics of the intervention expressed as mean values (range) were: $12 \mathrm{~min}$ of AOT for each session (9-18); 4 min for each video; 10.7 min of observed action performance (3-36); 5 sessions a week (3-7); the total duration of treatment $=46.7$ days (9-180 days). In all studies, the control group performed the same actions as the experimental group for the same amount of time. All eight studies administered AOT through goal-directed videos with specific hand/arm motor skill contents and afterward, the participants were asked to execute the same action. ${ }^{18,20,30-34}$ Only one study involved home-based AOT with repeated practice, ${ }^{35}$ and two studies conducted combining AOT with mCIMT. ${ }^{31,33}$

\section{Outcome Measures}

The outcomes of individual studies were extracted from the following: physical function and structures, activities, and participation. The primary outcome measures of this systematic review were Melbourne Assessment Scale, ABILHANDKids test, Box and Block Test (BBT), and Assisting Hand Assessment scale (AHA) to evaluate the upper limb function of children with hemiplegic CP. Furthermore, the ABILHAND-Kids test and Box and Block Test (BBT) were 


\begin{tabular}{|c|c|c|c|c|c|c|c|c|c|c|c|c|c|}
\hline 宅 & $\stackrel{0}{0}$ & $\check{\mathscr{E}}$ & $\stackrel{0}{z}$ & $\stackrel{\mathscr{u}}{\check{U}}$ & Z & zo & 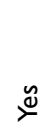 & $\stackrel{0}{z}$ & $\stackrel{\tilde{\nu}}{\tau}$ & $\stackrel{\mathscr{U}}{\longleftarrow}$ & 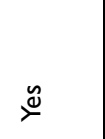 & $\frac{0}{6}$ & 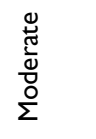 \\
\hline 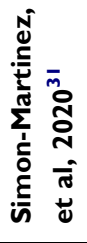 & $\stackrel{\mathscr{\nu}}{\check{0}}$ & 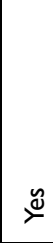 & $\stackrel{\circ}{z}$ & 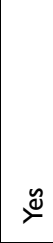 & z & zे & 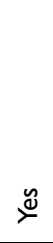 & $\stackrel{0}{z}$ & $\stackrel{\tilde{\nu}}{\tau}$ & 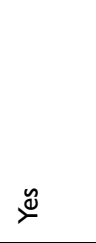 & $\stackrel{\varpi}{\simeq}$ & $\frac{\circ}{6}$ & 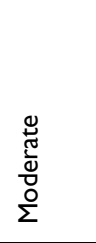 \\
\hline 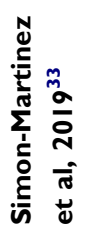 & 울 & $\stackrel{\mathscr{\Xi}}{\check{\infty}}$ & $\stackrel{0}{z}$ & 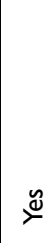 & zo & $\stackrel{0}{z}$ & 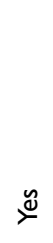 & $\stackrel{\circ}{z}$ & $\stackrel{\check{ٌ}}{\sim}$ & 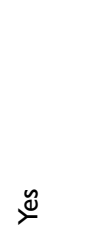 & $\stackrel{\check{\varpi}}{\tau}$ & $\frac{\circ}{6}$ & 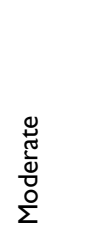 \\
\hline 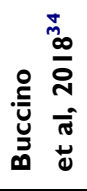 & $\stackrel{0}{\check{0}}$ & 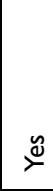 & $\stackrel{\circ}{z}$ & $\stackrel{\mathscr{u}}{.}$ & $\stackrel{\check{\Xi}}{\tau}$ & zo & $\stackrel{0}{z}$ & $\check{\beth}^{\mathscr{0}}$ & $\stackrel{\tilde{\nu}}{\check{\nu}}$ & $\underbrace{\tilde{u}}$ & 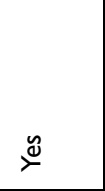 & $\stackrel{\circ}{\Sigma}$ & 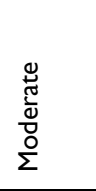 \\
\hline 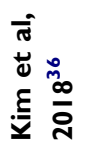 & 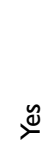 & $\stackrel{\mathscr{y}}{x}$ & $\stackrel{\circ}{z}$ & $\underbrace{y}$ & zo & $\stackrel{o}{z}$ & $\underbrace{\mathscr{y}}_{\check{y}}$ & $\stackrel{\mathscr{E}}{\approx}$ & $\stackrel{\tilde{y}}{\tau}$ & $\stackrel{\mathscr{\Xi}}{:}$ & $\stackrel{\mathscr{y}}{\tau}$ & $\stackrel{\circ}{\aleph}$ & 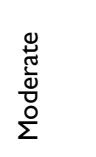 \\
\hline 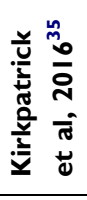 & $\stackrel{\check{\nu}}{\check{0}}$ & $\stackrel{\mathscr{N}}{\check{0}}$ & 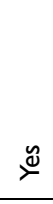 & $\stackrel{y}{\nu}$ & zo & $\stackrel{o}{z}$ & $\underbrace{\mathscr{y}}_{\check{U}}$ & $\stackrel{\mathscr{u}}{\tau}$ & $\stackrel{\tilde{u}}{\tau}$ & $\stackrel{\mathscr{\Xi}}{=}$ & $\stackrel{\mathscr{u}}{\succ}$ & $\stackrel{\circ}{\infty}$ & 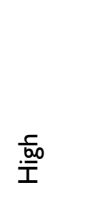 \\
\hline 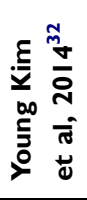 & 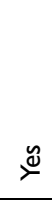 & $\stackrel{\mathscr{E}}{\check{E}}$ & $\stackrel{\circ}{z}$ & $\stackrel{y}{\nu}$ & zo & $\stackrel{o}{z}$ & $\stackrel{\mathscr{y}}{=}$ & $\stackrel{\circ}{z}$ & $\stackrel{\tilde{u}}{\tau}$ & $\stackrel{\mathscr{g}}{=}$ & $\stackrel{\mathscr{u}}{\tau}$ & $\frac{0}{6}$ & 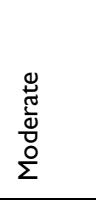 \\
\hline 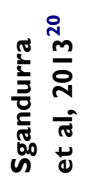 & $\stackrel{\circ}{z}$ & $\mathscr{s}^{\mathscr{x}}$ & $\stackrel{\mathscr{0}}{0}$ & $\stackrel{y}{\nu}$ & zo & zo & $\underbrace{\mathscr{y}}$ & $\stackrel{\check{s}}{\tau}$ & $\stackrel{\check{s}}{\tau}$ & $\stackrel{\mathscr{\Xi}}{\bullet}$ & $\stackrel{\check{\nu}}{\tau}$ & $\frac{\circ}{\infty}$ & $\frac{\text { 亳 }}{\underline{I}}$ \\
\hline 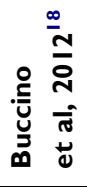 & $\stackrel{\mathscr{0}}{\check{0}}$ & $\stackrel{\mathscr{s}}{\check{\Delta}}$ & $\stackrel{\circ}{z}$ & $\stackrel{\mathscr{u}}{*}$ & $\stackrel{\mathscr{0}}{*}$ & $\stackrel{o}{z}$ & 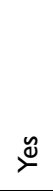 & $\stackrel{y}{\check{\nu}}$ & $\stackrel{\Xi}{\check{0}}$ & $\stackrel{\mathscr{y}}{=}$ & $\check{\nu}^{\mathscr{y}}$ & $\frac{\circ}{\infty}$ & $\frac{\text { 亳 }}{\underline{I}}$ \\
\hline 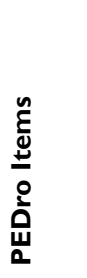 & 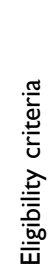 & 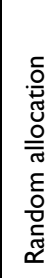 & 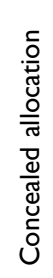 & 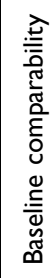 & 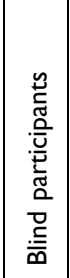 & 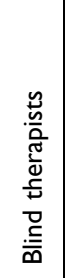 & 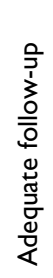 & 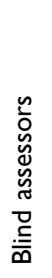 & 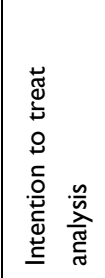 & 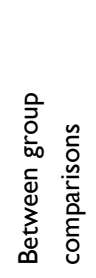 & 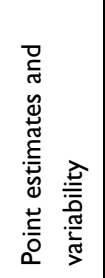 & 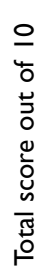 & 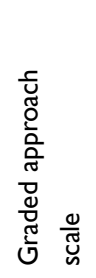 \\
\hline
\end{tabular}




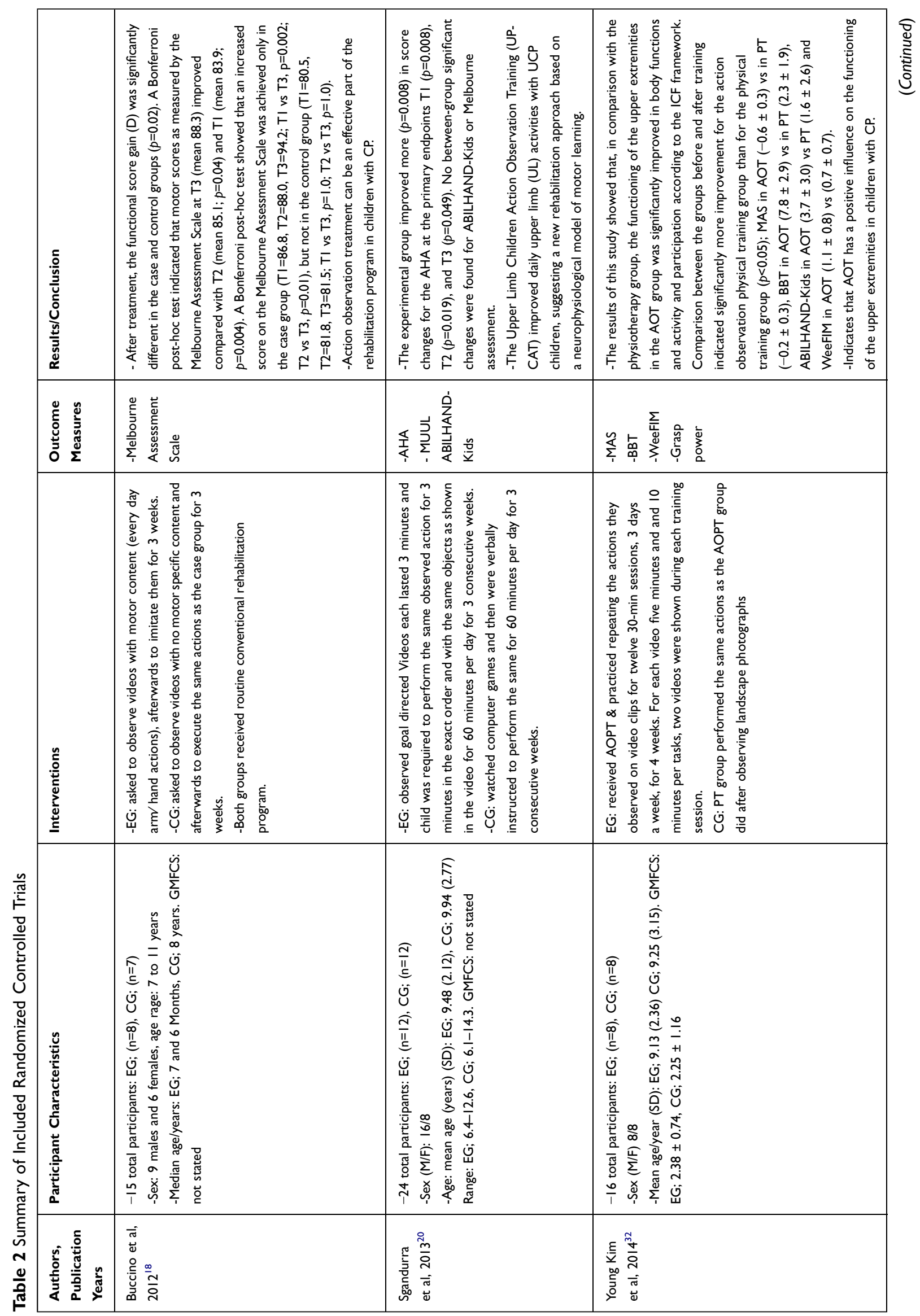




\begin{tabular}{|c|c|c|c|}
\hline & 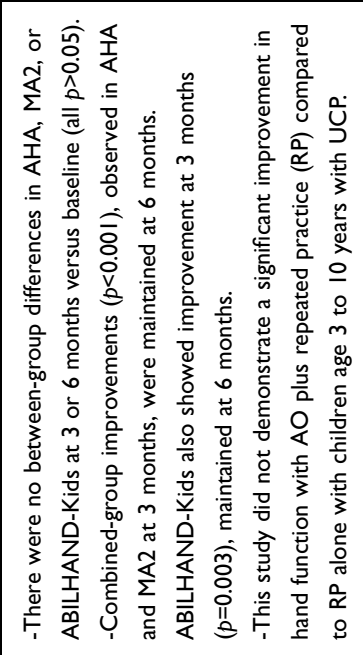 & 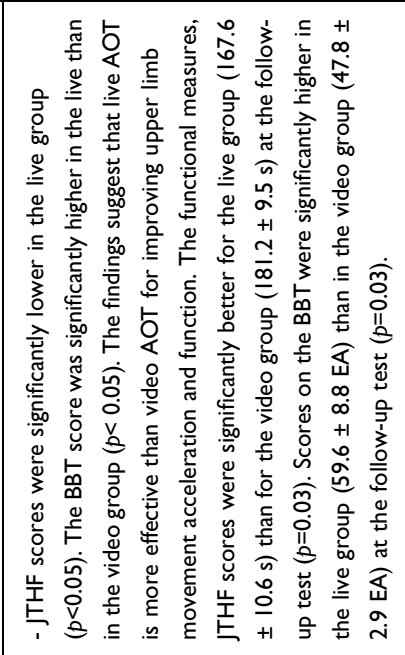 & 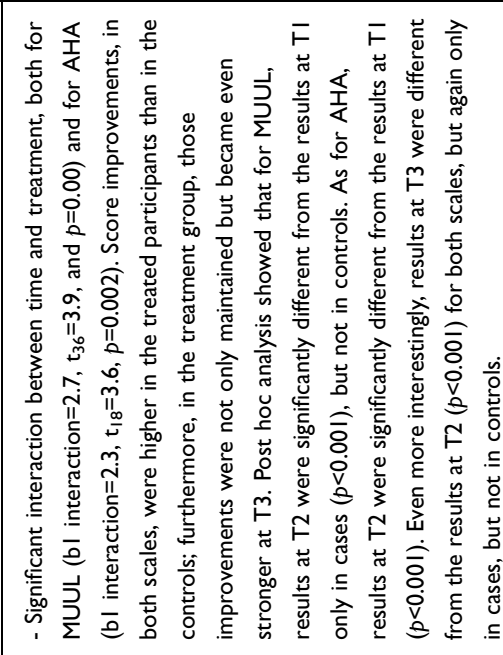 \\
\hline 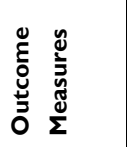 & 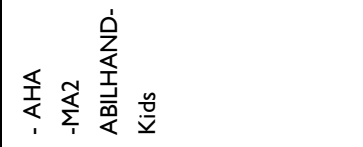 & 䓀占 & 志至 \\
\hline & 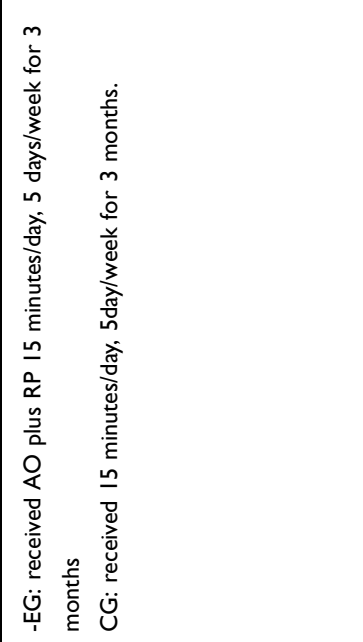 & 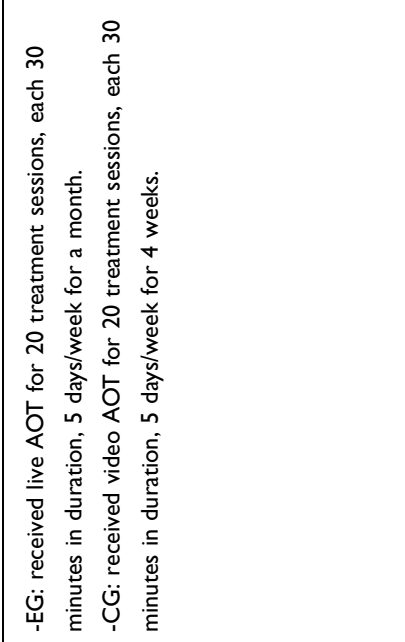 & 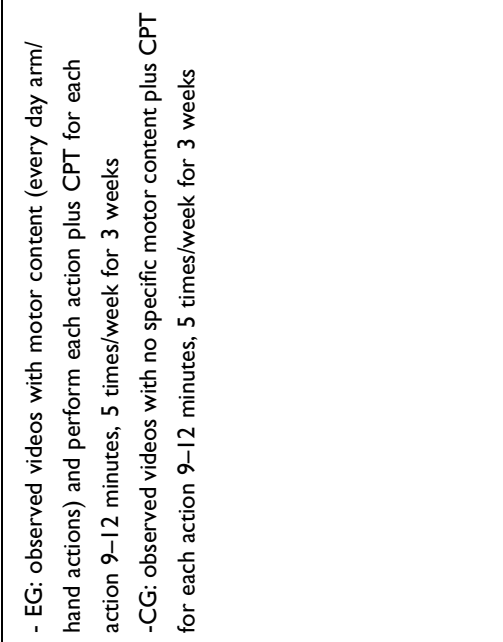 \\
\hline 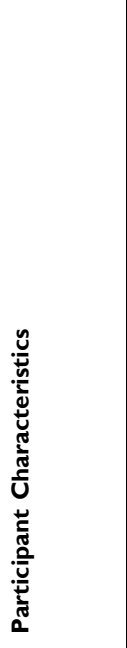 & 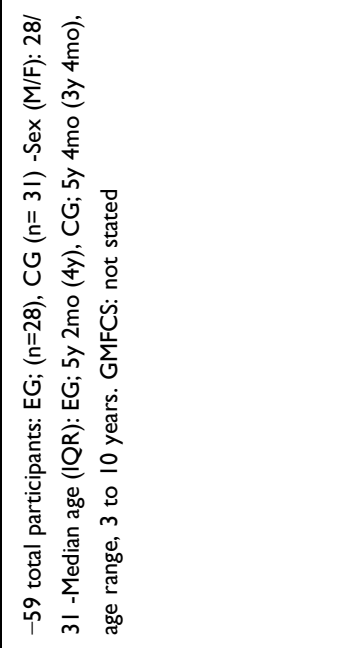 & 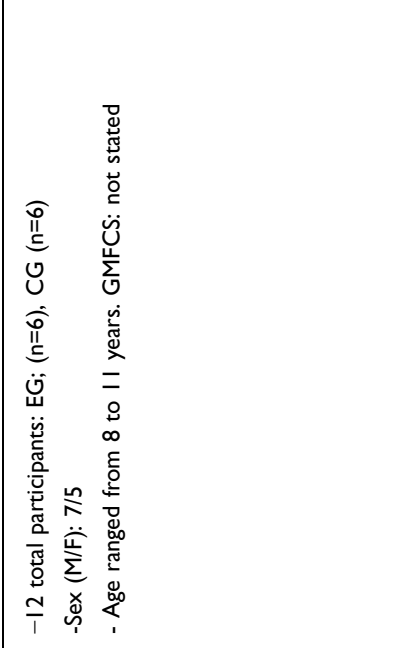 & 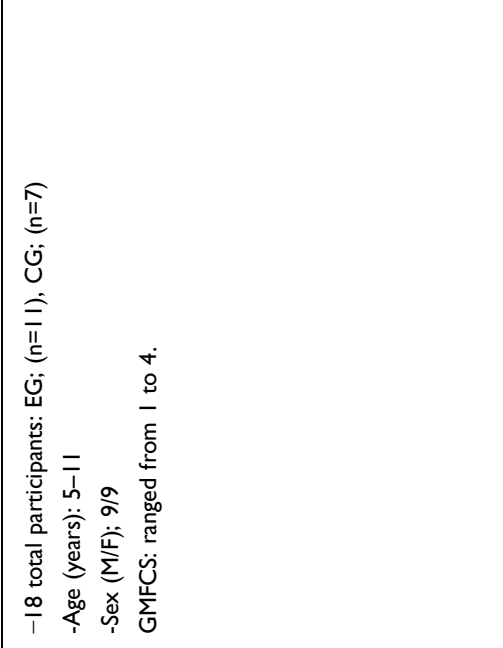 \\
\hline 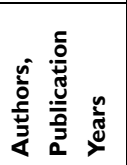 & 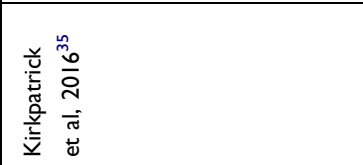 & 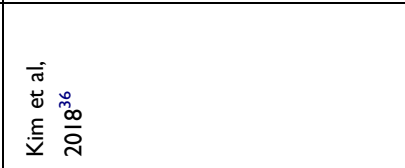 & 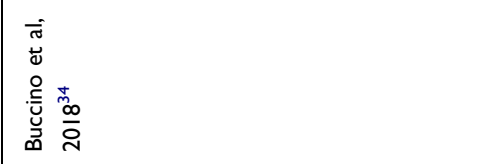 \\
\hline
\end{tabular}




\begin{tabular}{|c|c|}
\hline 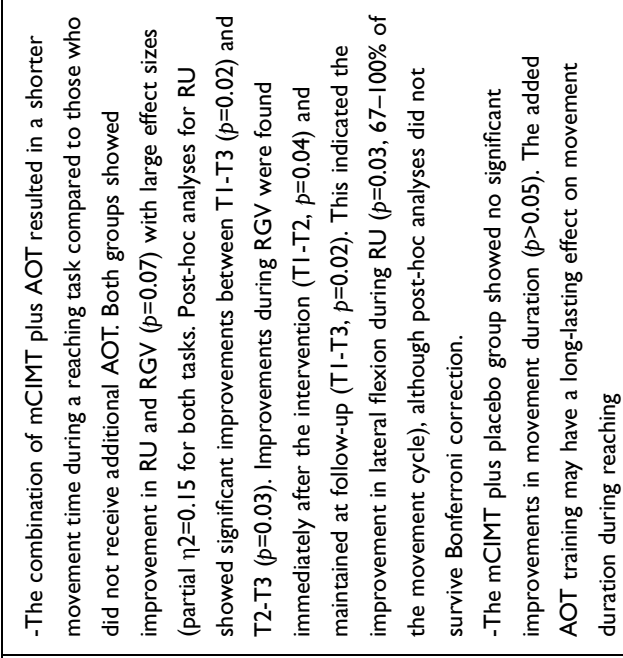 & 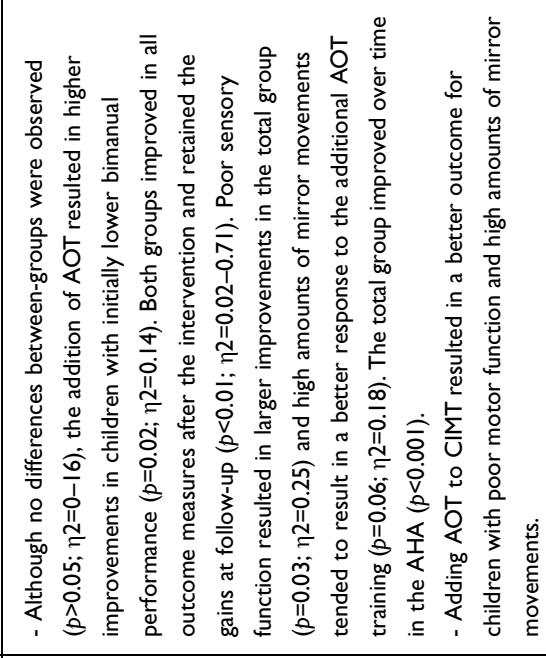 \\
\hline 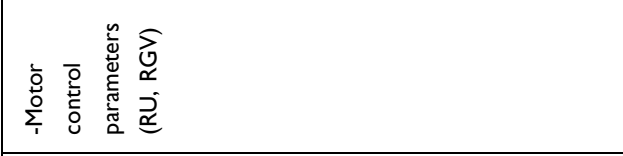 & 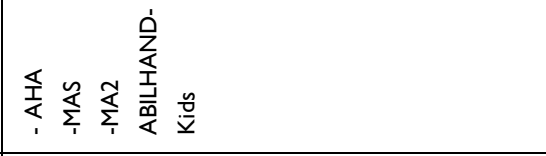 \\
\hline 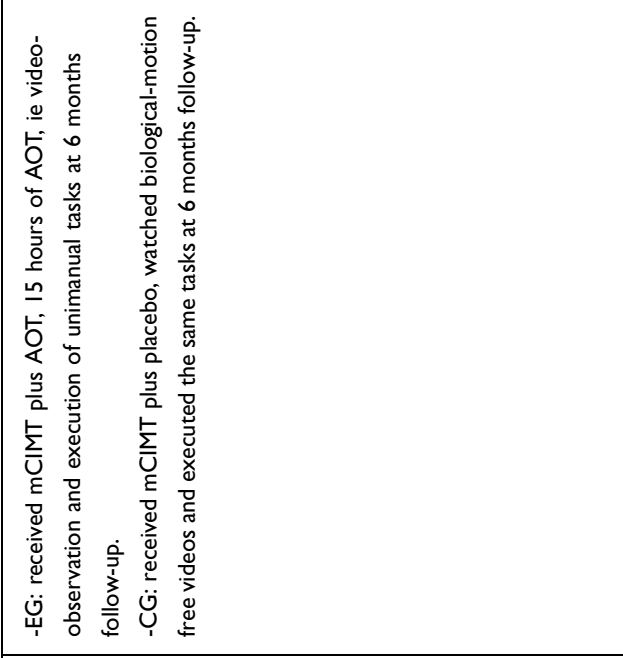 & 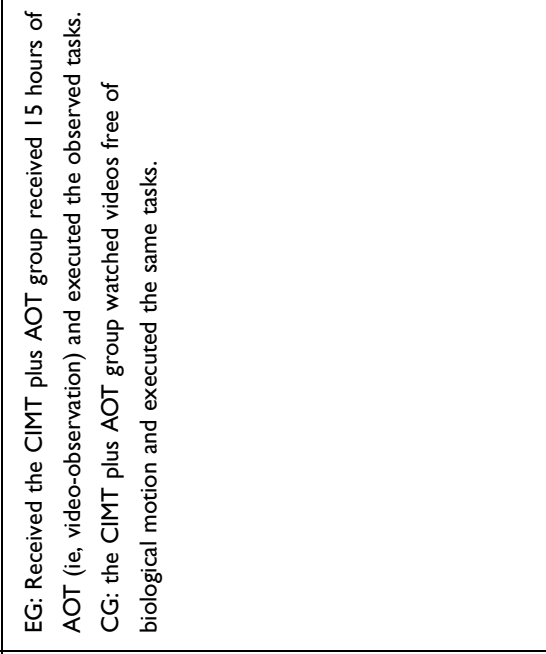 \\
\hline 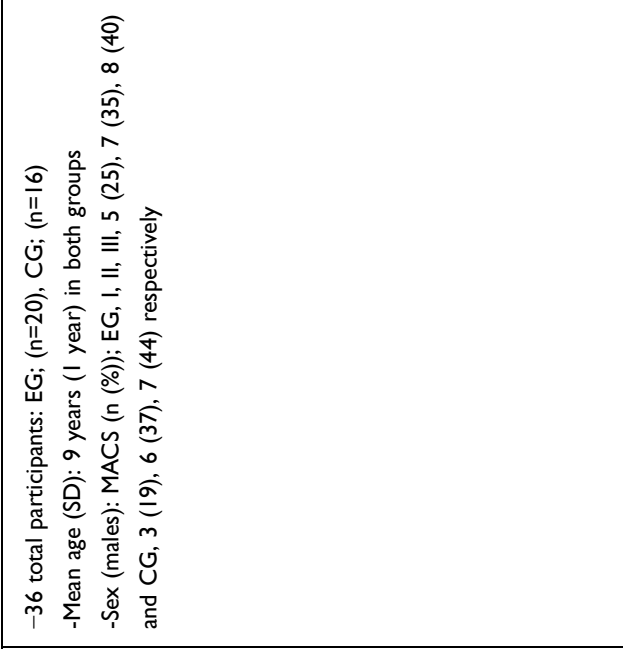 & 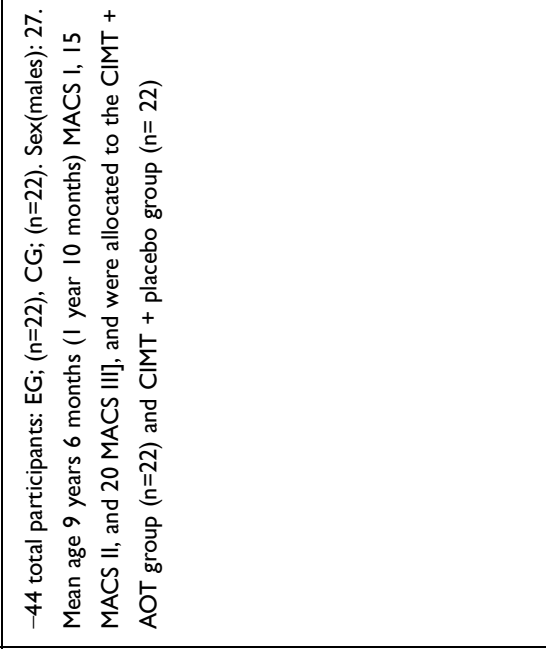 \\
\hline 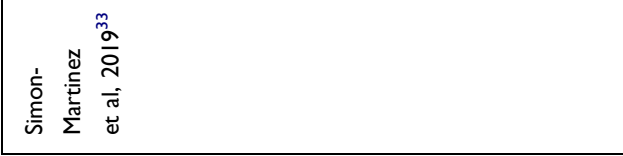 & 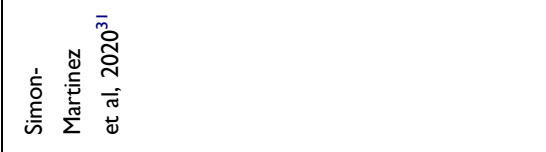 \\
\hline
\end{tabular}




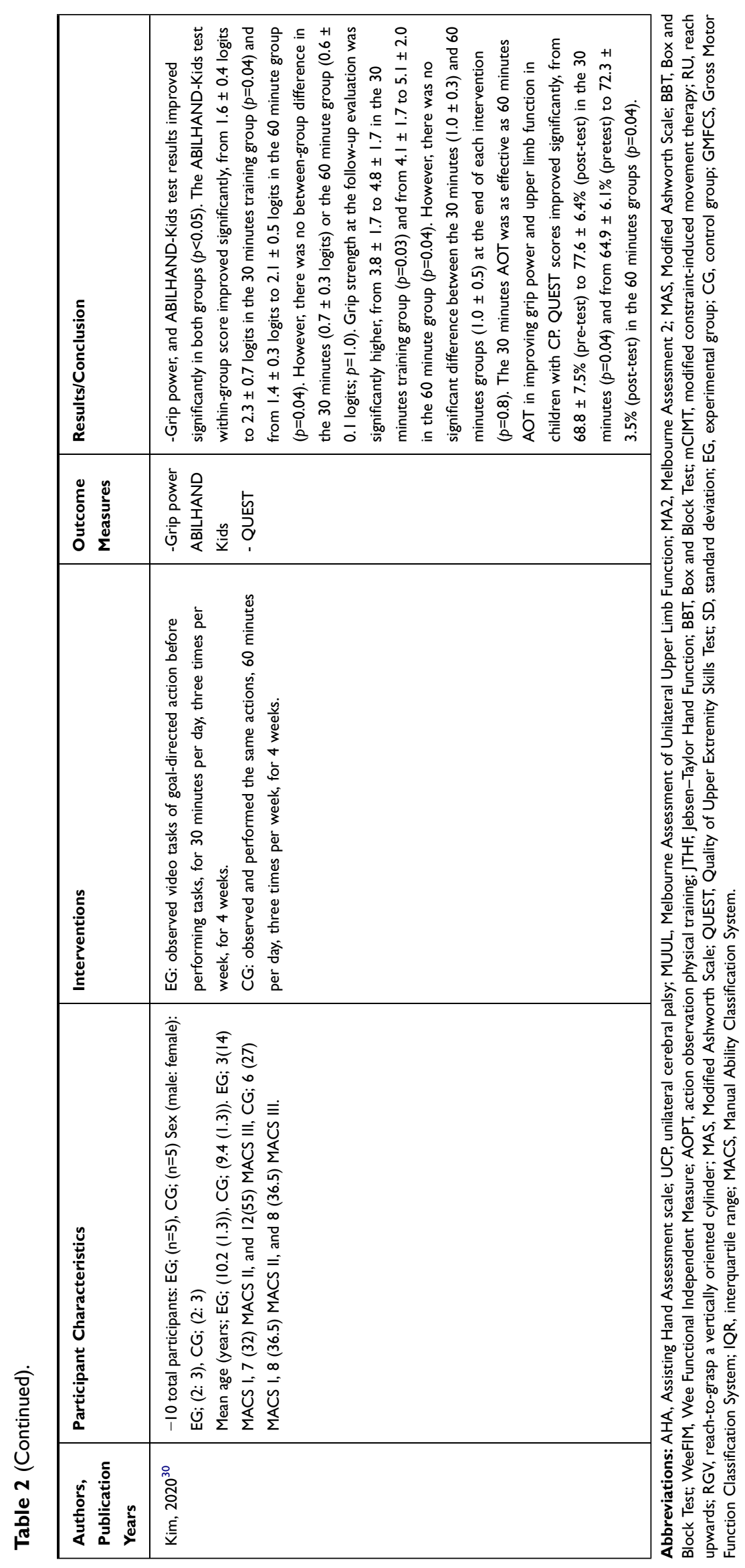




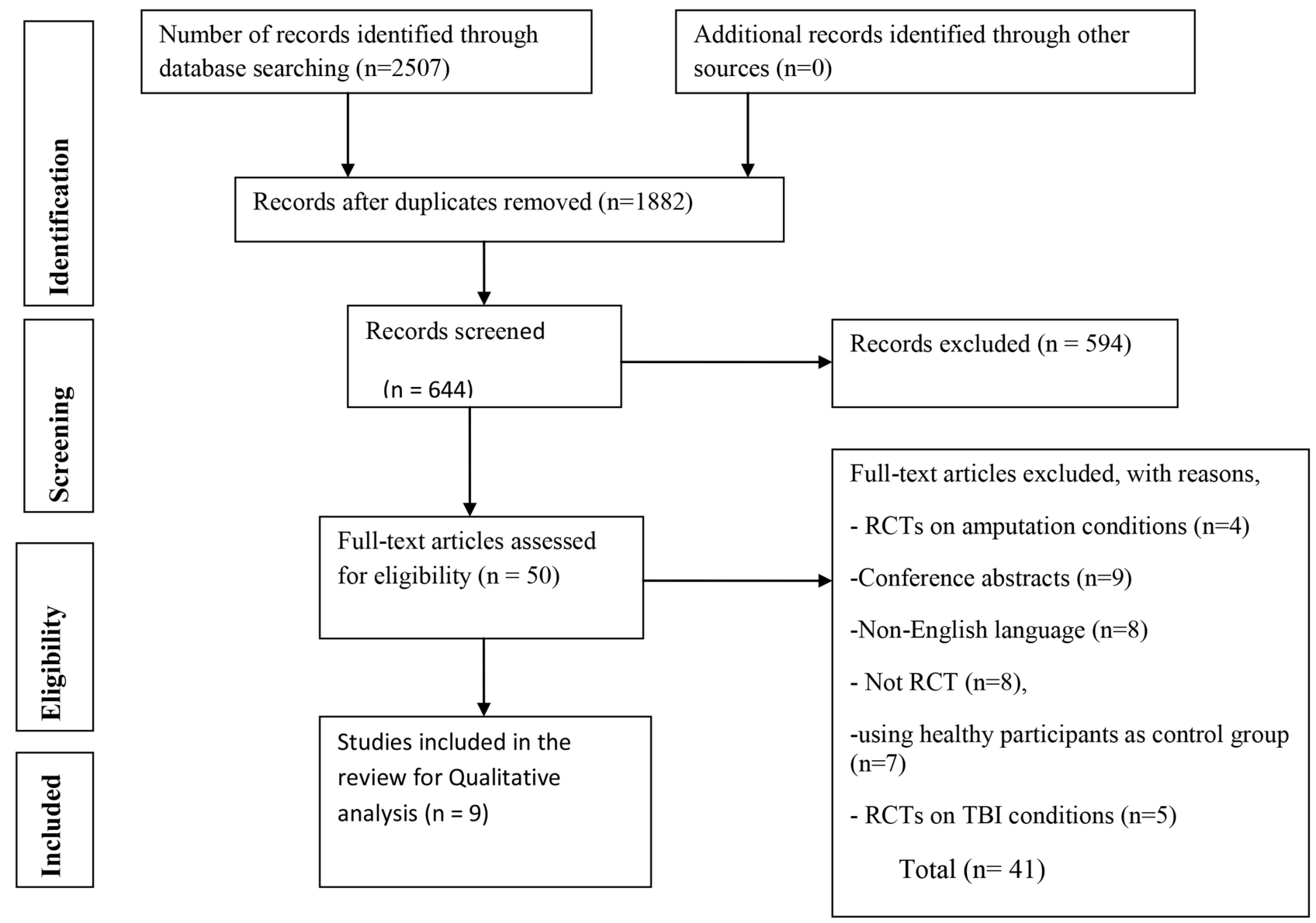

Figure I Preferred Reporting Items for Systematic Reviews and Meta-Analyses (PRISMA) diagram.Note: Adapted from Moher D, Liberati A, Tetzlaff J, Altman DG, The PRISMA Group (2009). Preferred Reporting Items for Systematic Reviews and Meta-Analyses: The PRISMA Statement. PLoS Med. 6(7): el000097. ${ }^{37}$

used to determine the subjects' activities and participation. The secondary outcome measures of this review were WeeFIM, QUEST, and motor control parameters. Two trials utilized WeeFIM and QUEST, designed to evaluate functional independence, movement patterns, and hand function. ${ }^{30,32}$ Five studies used the ABILHAND-Kids test to assess manual ability in children with $\mathrm{CP},{ }^{19,30,31,33,35}$ and two studies used BBT ${ }^{32,36}$ Five studies used the Melbourne Assessment Scale to evaluate the physical function and structure of children with UCP. $^{18,20,33-35}$ Similarly, another four studies performed a measure of upper limb motor function by using AHA. ${ }^{20,33-35}$

\section{Risk of Bias Across Studies}

Description of risk of bias within the individual studies and their rating is provided in Table 1. The PEDro score for all the included studies ranged from 6 to 8 , with a mean score of 7 . The quality assessment was performed in all included studies and the quality of evidence ranged between moderate to high. All included studies evaluated random allocation, baseline comparability, intention to treat analysis, point estimate, and variability, betweengroup comparisons. Only two studies ${ }^{20,35}$ assessed concealed allocation and, except one study, ${ }^{31}$ all included trials did not blind the therapist. The most prominent potential source of bias was related to blinding.

\section{Effects of AOT on Body Function and Structure}

Detailed extracted data on physical function and structure, from each trial, has been summarized and presented in Table 2. Nine studies, with 234 individuals, supplied published treatment effects on the upper limb physical function and structure of children with UCP. Out of the nine trials, eight of them $(n=175)$ confirmed that the body function of $\mathrm{CP}$ children was significantly improved in AOT groups as compared to the control groups in all outcome measures (AHA, MA2). ${ }^{18,20,30-34,36}$ However, only one $\operatorname{study}^{35}(\mathrm{n}=59)$ reported the action observation 
therapy had no significant improvement on the upper limb motor function compared to that of the control groups. Within the experimental intervention group, considerable improvement was found in upper limb function recovery in both outcome measures (AHA and MA2) at 3 month tests.

\section{Effects AOT on Activities and}

\section{Participation}

Out of included studies, seven of them, with 201 individuals with unilateral cerebral palsy proved that AOT has beneficial effects on the activities and participation. Their activities and participation were measured by using the ABILHAND-Kids test, BBT, WeeFIM, and QUEST whereby all outcome measures indicated a significant improvement in the experimental groups compared with the control groups in the activities and participation. ${ }^{20,30-32,36}$

\section{Adverse Effect}

The adverse effect of this intervention was not described in any included studies.

\section{Discussion}

The primary intention of this systematic review was to synthesize and examine the effectiveness of action observation therapy on upper limb rehabilitation in children with hemiplegic CP. To this point, there had been no reviews that determine the effectiveness of the action observation therapy on upper limb function in children with hemiplegic $\mathrm{CP}$ in a systematic way. Interestingly, most of the included trials with moderate to high quality of evidence confirmed that AOT was effective on upper limb motor function for children with hemiplegic CP. However, all included studies did not blind the assessor, which leads to assessor bias and could affect the estimation of the treatment effect. The overall effect of AOT on children with hemiplegic CP survivors was evaluated among included studies. Its overall effects were assessed on the different durations of intervention in children with UCP. The effect of AOT on physical function and structure was evaluated in all nine included studies. Likewise, of them seven studies evaluated AOT effects on activities and participation in subjects with hemiplegic CP. Eight studies confirmed that using AOT helps to improve physical function and structure, activities, and participation in children with hemiplegic $\mathrm{CP}^{18,20,30-34,36}$ However, in one study, AOT was not effective in upper limb motor function improvement. ${ }^{35}$ For instance, the study conducted by Sgandurra et $\mathrm{al}^{20}$ reported that AOT plus execution is more effective than execution alone in children with $\mathrm{CP}$. Especially, the AHA scale and ABILHAND-Kids scores differed significantly between the experimental and control groups. Similarly, Buccino et $\mathrm{al}^{18}$ observed the effects of AOT by comparing action practice after action observation and action practice alone in 15 children with $\mathrm{CP}$ (twelve hemiplegic and three with tetraplegia). They found that the mean Melbourne Assessment score increased significantly, from $86.87 \%$ to $94.25 \%$ in the AOT group. Conversely, Kirkpatrick et $\mathrm{al}^{35}$ found that home-based AOT and repeated practice did not improve the function of the upper limb more than repeated practice alone in children with CP. These possible differences could be because low-intensity upper limb therapy can be delivered at home by parents, and children in the experimental group may not always have observed movements before performing them, because of poor attention or lack of parental emphasis. Nevertheless, a study done by Kim et $\mathrm{al}^{32}$ indicates that significant improvement was observed in upper limb motor functions in children with cerebral palsy on MAS, BBT, and ABILHAND-Kids in response to action observation physical training compared to simple physical training. This might be due to AOT considering motor imitation characteristics of the mirror neuron system (MNS) can positively affect neural rehabilitation that is related to enormous range neural networks, ${ }^{38}$ and their changes enhance the potential to perform activities. ${ }^{39}$ Despite this fact, there were no observed significant differences in WeeFIM between experimental and control groups. This could be due to the troublesome effect of measurement tools in the forms of interviews and questionnaires, which leads to the sensitivity of subjects, and could not be evaluated easily.

The study done by Kim et $\mathrm{al}^{36}$ confirmed that live AOT is more effective than video AOT for improving UL movement acceleration and function. In addition, Buccino et $\mathrm{al}^{34}$ evidenced that AOT plus execution is more effective than execution alone in children with CP. They found improved upper limb motor function significantly in both scales (MUUL \& AHA) compared to the control groups. The possible explanation is that the brain target of AOT is exactly in the hand motor area, possibly involved in executing actions as well as in their processing which helps individuals to code for the motor properties of objects and the implementation of the most appropriate actions to act upon objects. ${ }^{40}$

Simon-Martinez et $\mathrm{al}^{33}$ confirmed that a combination of mCIMT and AOT resulted in a shorter movement time during a reaching task compared to those who received $\mathrm{mCIMT}$ alone. This proves that the additional AOT intervention for hemiplegic $\mathrm{CP}$ children on upper limb motor function was more 
effective, as AOT may have facilitated the activation of their mirror neuron system through video observation. Similarly, Kim et $\mathrm{al}^{30}$ supported the beneficial effect of AOT in improving grip strength and upper limb function in children with hemiplegic CP. A study conducted by Simon-Martinez et $\mathrm{al}^{31}$ showed that the CIMT plus AOT group benefited more than the CIMT plus placebo group on the upper extremity function of children with $\mathrm{CP}$ and their daily living activities. However, AOT did not show an overall added effect on improving upper limb function in children with unilateral CP when combined with CIMT in an intensive training approach.

Taken as a whole, one study reported that AOT had no beneficial effect on upper limb motor function. Nevertheless, taking into account the results of eight studies, a more plausible explanation proved that AOT had a significant improvement on grip power, hand function, activities, and participation in activity of daily living in hemiplegic CP children in comparison to the control groups.

\section{Limitations}

This review had the following limitations: it included only English language articles. Hence, there might be a chance of missing articles published in non-English languages. Most of the include studies were not blinded to the assessor, which can lead to bias estimates of treatment effect. Due to the heterogeneity of included studies, a meta-analysis was not carried out.

\section{Clinical Implication}

The finding of this review suggested that AOT appears to result in improved outcomes on body function and structure, activities, and participation of children with CP. Medical choice-making should rely on the cognitive and perceptual ability of children concerning the age and accessibility of needed equipment for AOT in a resource-limited setting.

\section{Conclusion}

Taken together, these findings suggest action observation therapy was found to be a better rehabilitative intervention for upper limb rehabilitation to improve physical function and structure, activities, and participation of children with hemiplegic CP than simple motor training. However, considerable attention must be paid when we use AOT for CP children, due to the severity of motor impairment, and cognitive status; further studies are needed to determine the optimal frequency, intensity, and time of AOT on these particular study subjects.

\section{Ethical Approval}

Ethical approval or patient consent were not required, since the present study was a review of previously published literature.

\section{Author Contributions}

All authors have contributed to the work reported, in the conception, study design, execution, acquisition of data, analysis and interpretation, have drafted, or substantially revised or critically reviewed the article, agreed on the journal to which the article is to be submitted, checked and agreed on all versions of the article before submission, during revision. Finally, any significant changes introduced at the proofing stage, agree to take responsibility and be accountable for the contents of the article.

\section{Funding}

There is no funding to report.

\section{Disclosure}

The authors report no conflicts of interest for this work.

\section{References}

1. Bax M, Goldstein M, Rosenbaum P, et al. Proposed definition and classification of cerebral palsy, April 2005. Dev Med Child Neurol. 2005;47(8):571-576. doi:10.1017/S001216220500112X

2. Ferrari A, Cioni G. The Spastic Forms of Cerebral Palsy: A Guide to the Assessment of Adaptive Functions. Springer; 2009.

3. Oskoui M, Coutinho F, Dykeman J, et al. An update on the prevalence of cerebral palsy: a systematic review and meta-analysis. Dev Med Child Neurol. 2013;55(6):509-519. doi:10.1111/dmcn.12080

4. Rosenbaum P, Gorter JW, Palisano R, et al. The relationship of cerebral palsy subtype and functional motor impairment: a population-based study. Dev Med Child Neurol. 2010;52 (7):682-683. doi:10.1111/j.1469-8749.2010.03652.x

5. White DA, Christ SE. Executive control of learning and memory in children with bilateral spastic cerebral palsy. J Int Neuropsychol Soc. 2005;11(7):920-924. doi:10.1017/S1355617705051064

6. Steenbergen B, Gordon AM. Activity limitation in hemiplegic cerebral palsy: evidence for disorders in motor planning. Dev Med Child Neurol. 2006;48(9):780-783. doi:10.1017/S0012162206001666

7. Uvebrant P. Hemiplegic cerebral palsy aetiology and outcome. Acta Paediatr. 1988;77:1-100.

8. Gordon AM, Hung Y-C, Brandao M, et al. Bimanual training and constraint-induced movement therapy in children with hemiplegic cerebral palsy: a randomized trial. Neurorehabil Neural Repair. 2011;25(8):692-702. doi:10.1177/1545968311402508

9. Sakzewski L, Gordon A, Eliasson A-C. The state of the evidence for intensive upper limb therapy approaches for children with unilateral cerebral palsy. J Child Neurol. 2014;29(8):1077-1090. doi:10.1177/ 0883073814533150

10. Hoare B, Imms C, Villanueva E, et al. Intensive therapy following upper limb botulinum toxin A injection in young children with unilateral cerebral palsy: a randomized trial. Dev Med Child Neurol. 2013;55(3):238-247. doi:10.1111/dmcn.12054 
11. Sarasso E, Gemma M, Agosta F, et al. Action observation training to improve motor function recovery: a systematic review. Arch Physiother. 2015;5(1):14. doi:10.1186/s40945-015-0013-x

12. Zhu M-H, Wang J, Gu X-D, et al. Effect of action observation therapy on daily activities and motor recovery in stroke patients. Int J Nurs Stud. 2015;2(3):279-282. doi:10.1016/j.ijnss.2015.08.006

13. Buccino G, Binkofski F, Fink GR, et al. Action observation activates premotor and parietal areas in a somatotopic manner: an fMRI study. Soc Neurosci. 2013;133-142.

14. Dinomais M, Lignon G, Chinier E, et al. Effect of observation of simple hand movement on brain activations in patients with unilateral cerebral palsy: an fMRI study. Res Dev Disabil. 2013;34 (6):1928-1937. doi:10.1016/j.ridd.2013.03.020

15. Rizzolatti G, Fadiga L, Gallese V, et al. Premotor cortex and the recognition of motor actions. Cogn Brain Res. 1996;3(2):131-141. doi:10.1016/0926-6410(95)00038-0

16. Buccino G, Binkofski F, Fink GR, et al. Action observation activates premotor and parietal areas in a somatotopic manner: an fMRI study. Eur J Neurosci. 2001;13(2):400-404.

17. Sgandurra G, Biagi L, Fogassi L, et al. Reorganization of the action observation network and sensory-motor system in children with unilateral cerebral palsy: an fMRI study. Neural Plast. 2018;2018:1-15. doi:10.1155/2018/6950547

18. Buccino G, Arisi D, Gough P, et al. Improving upper limb motor functions through action observation treatment: a pilot study in children with cerebral palsy. Dev Med Child Neurol. 2012;54 (9):822-828. doi:10.1111/j.1469-8749.2012.04334.x

19. Sgandurra G, Ferrari A, Cossu G, et al. Upper limb children action-observation training (UP-CAT): a randomised controlled trial in hemiplegic cerebral palsy. BMC Neurol. 2011;11(1):80. doi:10.1186/1471-2377-11-80

20. Sgandurra G, Ferrari A, Cossu G, et al. Randomized trial of observation and execution of upper extremity actions versus action alone in children with unilateral cerebral palsy. Neurorehabil Neural Repair. 2013;27(9):808-815. doi:10.1177/1545968313497101

21. Borges LR, Fernandes AB, Melo LP, et al. Action observation for upper limb rehabilitation after stroke. Cochrane Database Syst Rev. 2018;10.

22. Buchignani B, Beani E, Pomeroy V, et al. Action observation training for rehabilitation in brain injuries: a systematic review and meta-analysis. BMC Neurol. 2019;19(1):344. doi:10.1186/s12883019-1533-x

23. Peng T-H, Zhu J-D, Chen -C-C, et al. Action observation therapy for improving arm function, walking ability, and daily activity performance after stroke: a systematic review and meta-analysis. Clin Rehabil. 2019;33(8):1277-1285. doi:10.1177/0269215519839108

24. Thieme H, Morkisch N, Rietz C, et al. The efficacy of movement representation techniques for treatment of limb pain - a systematic review and meta-analysis. J Pain. 2016;17(2):167-180. doi:10.1016/ j.jpain.2015.10.015

25. Caligiore D, Mustile M, Spalletta G, et al. Action observation and motor imagery for rehabilitation in Parkinson's disease: a systematic review and an integrative hypothesis. Neurosci Biobehav Rev. 2017;72:210-222. doi:10.1016/j.neubiorev.2016.11.005
26. Moher D, Shamseer L, Clarke M, et al. Preferred reporting items for systematic review and meta-analysis protocols (PRISMA-P) 2015 statement. Syst Rev. 2015;4(1):1. doi:10.1186/2046-4053-4-1

27. Maher CG, Sherrington C, Herbert RD, et al. Reliability of the PEDro scale for rating quality of randomized controlled trials. Phys Ther. 2003;83(8):713-721. doi:10.1093/ptj/83.8.713

28. de Morton NA. The PEDro scale is a valid measure of the methodological quality of clinical trials: a demographic study. Aust $J$ Physiother. 2009;55(2):129-133. doi:10.1016/S0004-9514(09)70043-1

29. Guyatt GH, Oxman AD, Kunz R, et al. GRADE guidelines: 7. Rating the quality of evidence-inconsistency. J Clin Epidemiol. 2011;64 (12):1294-1302. doi:10.1016/j.jclinepi.2011.03.017

30. Kim DH. Comparison of short-and long-time action observation training (AOT) on upper limb function in children with cerebral palsy. Physiother Pract Res. (Preprint):1-6.

31. Simon-Martinez C, Mailleux L, Hoskens J, et al. Randomized controlled trial combining constraint-induced movement therapy and action-observation training in unilateral cerebral palsy: clinical effects and influencing factors of treatment response. Ther $A d v$ Neurol Disord. 2020;13:1756286419898065. doi:10.1177/ 1756286419898065

32. Kim JY, Kim J-M, Ko EY. The effect of the action observation physical training on the upper extremity function in children with cerebral palsy. J Exerc Rehabil. 2014;10(3):176. doi:10.12965/ jer.140114

33. Simon-Martinez C, Mailleux L, Jaspers E, et al. Effects of combining constraint-induced movement therapy and action-observation training on upper limb kinematics in children with unilateral cerebral palsy: a randomized controlled trial. Sci Rep. 2020;10:1-15.

34. Buccino G, Molinaro A, Ambrosi C, et al. Action observation treatment improves upper limb motor functions in children with cerebral palsy: a combined clinical and brain imaging study. Neural Plast. 2018;2018:1-11. doi:10.1155/2018/4843985

35. Kirkpatrick E, Pearse J, James P, et al. Effect of parent-delivered action observation therapy on upper limb function in unilateral cerebral palsy: a randomized controlled trial. Dev Med Child Neurol. 2016;58(10):1049-1056. doi:10.1111/dmcn.13109

36. Kim D-H, An D-H, Yoo W-G. Effects of live and video form action observation training on upper limb function in children with hemiparetic cerebral palsy. Technol Health Care. 2018;26(3):437-443. doi:10.3233/THC-181220

37. Moher D, Liberati A, Tetzlaff J, Altman DG, The PRISMA Group (2009). Preferred reporting items for systematic reviews and metaanalyses: The PRISMA statement. PLoS Med. 6(7):e1000097.

38. Iacoboni M, Koski LM, Brass M, et al. Reafferent copies of imitated actions in the right superior temporal cortex. Proc Natl Acad Sci U S A. 2001;98(24):13995-13999. doi:10.1073/pnas.241474598

39. Buccino G, Solodkin A, Small SL. Functions of the mirror neuron system: implications for neurorehabilitation. Cogn Behav Neurol. 2006;19(1):55-63. doi:10.1097/00146965-200603000-00007

40. Biagi L, Cioni G, Fogassi L, et al. Action observation network in childhood: a comparative fMRI study with adults. Dev Sci. 2016;19 (6):1075-1086. doi:10.1111/desc. 12353
Pediatric Health, Medicine and Therapeutics

\section{Publish your work in this journal}

Pediatric Health, Medicine and Therapeutics is an international, peerreviewed, open access journal publishing original research, reports, editorials, reviews and commentaries. All aspects of health maintenance, preventative measures and disease treatment interventions are addressed within the journal. Practitioners from all disciplines are invited to submit their work as well as healthcare researchers and patient support groups. The manuscript management system is completely online and includes a very quick and fair peer-review system. Visit http://www.dovepress.com/testimonials.php to read real quotes from published authors. 